University of Nebraska - Lincoln

DigitalCommons@University of Nebraska - Lincoln

Virology Papers

Virology, Nebraska Center for

2005

\title{
Compartmentalization of HIV-1 in the Central Nervous System: Role of the Choroid Plexus
}

\author{
Evan J. Burkala \\ University of Nebraska-Lincoln \\ Jun $\mathrm{He}$ \\ University of Nebraska-Lincoln \\ John T. West \\ University of Nebraska-Lincoln, jwest2@unl.edu \\ Charles Wood \\ University of Nebraska-Lincoln, cwood1@unl.edu \\ Carol K. Petito \\ University of Miami School of Medicine
}

Follow this and additional works at: https://digitalcommons.unl.edu/virologypub

Part of the Virology Commons

Burkala, Evan J.; He, Jun; West, John T.; Wood, Charles; and Petito, Carol K., "Compartmentalization of HIV-1 in the Central Nervous System: Role of the Choroid Plexus" (2005). Virology Papers. 148.

https://digitalcommons.unl.edu/virologypub/148

This Article is brought to you for free and open access by the Virology, Nebraska Center for at DigitalCommons@University of Nebraska - Lincoln. It has been accepted for inclusion in Virology Papers by an authorized administrator of DigitalCommons@University of Nebraska - Lincoln. 
Originally published in AIDS 19 (2005): 675-684.

http:/ / www.aidsonline.com/ Copyright 2005 by Lippincott, Williams \& Wilkins.

Used by permission.

\title{
Compartmentalization of HIV-1 in the Central Nervous System: Role of the Choroid Plexus
}

\author{
Evan J. Burkala ${ }^{a}$, Jun Hea ${ }^{a}$, John T. Westa ${ }^{a}$, Charles Wood ${ }^{a}$ and Carol K. Petito ${ }^{b}$ \\ From the a Nebraska Center for Virology and the School of Biological Sciences, University of Nebraska, \\ Lincoln, NE, USA, and the ${ }^{b}$ Department of Pathology, University of Miami School of Medicine, Miami, FL, USA. \\ Correspondence to Charles Wood, cwood1@unl.edu.
}

Received: July 23, 2004; revised: January 5, 2005; accepted: February 9, 2005.

\begin{abstract}
Objectives: To determine the genetic and phenotypic composition of HIV-1 found in the choroid plexus $(\mathrm{CPx})$ and its relationship to virus in the brain and peripheral lymphoid tissue.

Design: Phenotypic and molecular comparisons of HIV-1 found in autopsy brain, CPx, and spleen tissues.

Methods: HIV-1 was co-cultured from matched postmortem brain (basal ganglia), CPx, and spleen tissues of AIDS patients with and without HIV-associated encephalitis and dementia. Viral phenotypes were determined by infection of monocyte-derived macrophages, MT-2 or co-receptor-specific cell lines. Viral env and pol sequences were determined from genomic DNA isolated directly from tissues or co-cultures, and phylogenetic comparisons were performed.

Results: CCR5-utilization was the most prevalent viral tropism found in all tissues, although spleen isolates also displayed CXCR4 usage. Viruses isolated from CPx consisted of both peripheral and brain-like virus, but were more related phenotypically and genetically to those found in the brain. Mutations found in the pol gene that could confer drug resistance to brain and $\mathrm{CPx}$ isolates were similar to those found in the periphery.

Conclusion: The CPx contained replication-competent virus that was most similar, although distinct, from that found in the brain. It also contained some viruses with high similarity to those of peripheral origin. Compartmentalization of viral env and pol sequences indicated that differential selective pressures exist in each tissue examined. These studies suggest that the CPx may provide an environment that promotes the evolution of drug-resistant strains with central nervous system tropism, although it is unlikely to be a reservoir for archival HIV-1 variants.
\end{abstract}

Keywords: brain, choroid plexus, compartmentalization, drug resistance, env, HIV-1, pol

\section{Introduction}

Morbidity caused by neurological complications of HIV-1 infection has become increasingly common since the implementation of highly active antiretroviral therapy (HAART), as a result of the prolonged life expectancy of infected individuals. The events leading to HIV-1 brain infection and progression to HIV-1-associated dementia
(HAD) are not understood. HIV-1 enters the central nervous system (CNS) early during primary infection and can be isolated from cerebrospinal fluid (CSF) at this time [1]; however, viral replication in the brain is rare before the onset of immunosuppression and AIDS. Once infection of the brain has been established, the virus appears to undergo independent micro-evolution or com- 
partmentalization, an effect commonly observed in other tissues [2-5]. Compartmentalization probably has clinical implications on therapy by reducing the effectiveness and durability of drug treatment and vaccination strategies by the sequestration of drug-resistant mutants and the evolution of neurotoxic quasispecies.

The choroid plexus (CPx), the main tissue involved in the secretion and regulation of CSF, may play a key role in the neuropathogenesis of HIVencephalitis (HIVE). The $\mathrm{CPx}$ consists of two fluid-cellular interfaces, one with capillary blood and one with CSF. The CPx endothelial cells express adhesion molecules that initiate lymphocyte migration in response to inflammation, but unlike the blood-brain barrier are not joined by tight junctions [68]. The epithelial layer of the CPx forms the blood-CSF barrier of the brain; these cells are joined by tight junctions and regulate the bidirectional entry of macromolecules, ions, cells, and perhaps virus, between the blood, CSF, and brain parenchyma [6,9]. The close proximity of readily permissive monocytes and T cells in the CPx stroma implies its involvement in the transmission of virus between the CNS and peripheral compartments [10].

Previous studies have suggested that the CPx is a site for viral dissemination into the CNS as the viral genotypes isolated from CPx tissues demonstrated similarity with those from the peripheral and CNS compartments $[11,12]$. However, such studies were limited to formalin-fixed paraffin-embedded tissues, and we were thus unable to study the properties of CPx-derived virus. Accordingly, in this study, we isolated viruses from autopsy tissue samples of brain, CPx, and spleen from three AIDS patients to characterize the viral genotypes and phenotypes found in these three tissue compartments. Our results confirm that the $\mathrm{CPx}$ contains a composite of viruses with properties similar to those found in both brain and spleen tissues.

\section{Methods}

\section{Case selection and tissue preparation}

All tissues were obtained with Internal Review Board approval from the University of Miami according to National Institutes of Health (NIH) guidelines. At autopsy, fresh brain was bisected in the mid-saggital plane and pieces of basal ganglia, frontal lobe and CPx from the trigone of the lateral ventricle were washed several times in phosphate-buffered saline to remove blood contamination, and were rapidly frozen at 708C. Spleen tissues were treated similarly. Additional tissue samples from HIV-1-infected patients and controls were donated by the National NeuroAIDS Tissue Consortium in Galveston, Texas, USA (MH59656) and the University of Miami Brain and Tissue Bank (N01 HD83284). Determination of HAD was based on records provided by the National
NeuroAIDS Tissue Consortium and American Academy of Neurology criteria using clinical records [13]. Neuropathological examination of brain tissue sections acquired at the University of Miami was performed using hematoxylin and eosin-stained sections.

\section{Virus Isolation}

Tissue samples were thawed, washed twice with phosphate-buffered saline, and ground through a $100 \mathrm{~mm}$ cell strainer (BD Bioscience, USA) with the addition of RPMI containing $10 \%$ fetal bovine serum (Nova-tech, NE, USA) to obtain a tissue homogenate. Phytohemagglutinin-stimulated peripheral blood mononuclear cell (PBMC) cultures containing $10 \mathrm{U} / \mathrm{ml}$ human IL-2 were inoculated with the cell suspension (Roche, Indianapolis, IN, USA). PBMC used in the study were obtained from a local blood bank and were a pool of PBMC from two to four anonymous donors. Cells were cultured for 5 days before replacing the media and supplementing with fresh uninfected phytohemagglutinin-stimulated PBMC. HIV-1 p24 antigen capture assays (Coulter, USA) of culture supernatants at 5,10, and 15 days post-infection confirmed the production of virus from tissue homogenate co-cultures. Cell-free culture supernatants collected 14-21 days post-infection were used to infect fresh PBMC. Genomic DNA was extracted from approximately $10^{7}$ first passage PBMC, or from tissue (50 mg) according to the manufacturer's protocol (Gentra, USA).

\section{Virus phenotypic analyses}

Co-receptor usage was determined by infecting each of the GHOST(3) chemokine receptor-expressing cell lines obtained from the NIH AIDS Reagent and Reference Reagent Program (i.e. CCR1, CCR2, CCR3, CCR4, CCR5, CCR8, CXCR4, CXCR6, CX3CR1, BOB, CCR3/CCR5/ CXCR4). Approximately 100 TCID50 of primary virus isolates (per $7.5 \mathrm{~mm}^{2}$ well) were used to infect each GHOST(3) cell line for $36 \mathrm{~h}$ before analysis by fluorescence microscopy. The co-receptor assay was performed three times independently, each in triplicate, and was scored qualitatively in comparison with the background observed for the chemokine receptor-negative GHOST(3) parental line.

Virus isolates were evaluated for syncytium formation by infection of MT-2 and C8166 T-cell lines. An assessment of productive infection of macrophages was performed with infection of adherent monocytes derived from PBMC. The culture supernatant was monitored for HIV-1 p24 production by antigen capture immunoassay (Coulter) for 15 days post-infection.

\section{Polymerase chain reaction and sequencing}

For all genetic analyses, DNA isolated from first passage co-cultures of tissues from the three virus-positive indi- 
Table 1. Characterization of incidence of HIV-1 neurological disorders and ability to detect and culture HIV from various tissues in the sample group.

\begin{tabular}{lccccccc}
\hline Disease status & No. cases & $\begin{array}{c}\text { Average age } \\
\text { (years } \pm \text { SD) }\end{array}$ & HIVE & HAD & PMI $(\mathrm{h} \pm \mathrm{SD})$ & Tissue & $\begin{array}{c}\text { Virus } \\
\text { PCR }(\%)\end{array}$ \\
isolation (\%)
\end{tabular}

BG, Basal ganglia; CPx, choroid plexus; FC, frontal cortex; FL, frontal lobe; HAD, HIV-associated dementia; HIVE, HIV encephalitis; n/a, information not available/applicable.

${ }^{a}$ Criteria for determination of HAD are described in the Materials and methods section.

Table 2. Phenotypic characterization of virus isolated from various tissues and the clinical data of these patients.

\begin{tabular}{|c|c|c|c|c|c|c|c|c|c|c|c|}
\hline Patient & Age & HAART & Dementia & Pathology & $\begin{array}{l}\text { Plasma } \\
\text { viral load }\end{array}$ & $\mathrm{CD} 4^{\mathrm{b}}$ & PMI & Tissue & $\begin{array}{l}\text { Co-receptor } \\
\text { usage }^{\text {c }}\end{array}$ & $\begin{array}{l}\text { Cell } \\
\text { tropism }^{\mathrm{d}}\end{array}$ & $\begin{array}{l}\text { Syncytium } \\
\text { formation }\end{array}$ \\
\hline \multirow[t]{3}{*}{ A } & \multirow[t]{3}{*}{45} & \multirow[t]{3}{*}{ Y } & \multirow[t]{3}{*}{$\mathrm{n} / \mathrm{a}$} & \multirow[t]{3}{*}{ HIVE } & \multirow[t]{3}{*}{28000} & \multirow[t]{3}{*}{14} & \multirow[t]{3}{*}{8.5} & Brain (BG) & CCR5, CCR3 & M & - \\
\hline & & & & & & & & $\mathrm{CPx}$ & $\begin{array}{l}\text { CCR5 (CCR3, } \\
\text { CCR4, CXCR4, } \\
\text { CX3CR1) }\end{array}$ & M & - \\
\hline & & & & & & & & Spleen & $\begin{array}{l}\text { CCR5, CXCR4, } \\
\text { CCR3, CCR8 } \\
\text { (CX3CR1, } \\
\text { CXCR6) }\end{array}$ & $M, T$ & + \\
\hline \multirow[t]{3}{*}{ B } & \multirow[t]{3}{*}{36} & \multirow[t]{3}{*}{$\mathrm{n} / \mathrm{a}$} & \multirow[t]{3}{*}{ Y } & \multirow[t]{3}{*}{ HIVE } & \multirow[t]{3}{*}{$\mathrm{n} / \mathrm{a}$} & \multirow[t]{3}{*}{4} & \multirow[t]{3}{*}{14.0} & Brain (BG) & $\begin{array}{c}\text { CCR5, CCR3 } \\
\text { (CCR4, } \\
\text { CXCR6) }\end{array}$ & M & - \\
\hline & & & & & & & & $\mathrm{CPx}$ & CCR5, CCR4 & M & - \\
\hline & & & & & & & & Spleen & CCR5, CXCR4 & $\mathrm{M}, \mathrm{T}$ & + \\
\hline \multirow[t]{3}{*}{ C } & \multirow[t]{3}{*}{71} & \multirow[t]{3}{*}{ Y } & \multirow[t]{3}{*}{ Y } & \multirow[t]{3}{*}{$\begin{array}{l}\text { Subcortical } \\
\text { arteriosclerotic } \\
\text { leukoencephalopathy } \\
\text { (Binswanger's disease) }\end{array}$} & \multirow[t]{3}{*}{103000} & \multirow[t]{3}{*}{49} & \multirow[t]{3}{*}{16.0} & Brain (BG) & $\begin{array}{l}\text { CCR5, CCR3 } \\
\text { (CCR2) }\end{array}$ & M & - \\
\hline & & & & & & & & $\mathrm{CPx}$ & $\begin{array}{c}\text { CCR5, CCR3 } \\
(\text { CXCR4) }\end{array}$ & M & - \\
\hline & & & & & & & & Spleen & $\begin{array}{c}\text { CCR5, CXCR4 } \\
(\text { CX3CR } 1)\end{array}$ & $M, T$ & + \\
\hline D & 32 & Y & Y & HIVE & 561321 & $\mathrm{n} / \mathrm{a}$ & 14.5 & $\begin{array}{l}\text { Brain (FL) } \\
\text { CPx } \\
\text { Spleen }\end{array}$ & & $\mathrm{n} / \mathrm{a}$ & \\
\hline E & 33 & Y & Y & HIVE, CMV & 2150000 & $\mathrm{n} / \mathrm{a}$ & 3 & $\begin{array}{l}\text { Brain }(F L) \\
\text { CPx } \\
\text { Spleen }\end{array}$ & & $\mathrm{n} / \mathrm{a}$ & \\
\hline
\end{tabular}

BG, Basal ganglia; CMV, cytomegalovirus; CPx, choroid plexus; FC, frontal cortex; FL, frontal lobe; HAART, highly active antiretroviral therapy; HIVE, HIV encephalitis; n/a, information not available/applicable; PMI, postmortem interval.

aplasma viral RNA load in copies/ $\mu$ l at last known collection date.

${ }^{\mathrm{b}} \mathrm{CD} 4$ cell count in cell $\mathrm{s} / \mathrm{mm}^{3}$ at last known collection date.

${ }^{\mathrm{C}}$ Co-receptor usage determined using GHOST(3) co-receptor expressing cell lines. Bold indicates primary usage of the co-receptor in the majority of replicates, normal indicates usage that was observed in less than $50 \%$ of replicates or at a notably reduced intensity, and parentheses () indicates minor usage that was infrequently observed in only few repeat experiments.

${ }^{\mathrm{d}}$ Tropism determined on MT-2 and C8166 cell lines, and primary monocyte-derived macrophages for T- and M-tropism, respectively. 
viduals plus DNA isolated directly from the three tissues of two virus-negative cases were used. For env gene analyses, proviral DNA was amplified using the ED3 and ED14 primers and ED5 and ED12 primers, in a nested reaction as previously described [14]. Amplification of pol sequences for resistance mutation analysis was performed using the JA203-206 set of nested primers [15]. When possible, first-round polymerase chain reaction (PCR) products were cloned using the pCR4-TOPO TA cloning vector (Invitrogen, USA). Cloned HIV-1 env sequences were screened by heteroduplex mobility assay (previously described by Delwart et al. [16]), and only those clones that were genetically distinct were sequenced using the ED31 primer to obtain the V3 region data. DNA sequences of cloned pol and env fragments (corresponding to 2135-3338 and 7017-7322 in HXB2, respectively) were determined and analysis was performed using the Vector NTI suite of programs (Version 9.0; InforMax, UK) and CLUSTALW. Potential N-linked glycosylation sites were identified using the N-glycosite program available on the HIV Sequence Database website (http:/ / www.hiv.lanl.gov/).

\section{Phylogenetic Analyses}

Phylogenetic and molecular evolutionary analyses were conducted using MEGA version 2.1 [17]. Estimations of genetic distance were made using MEGA with 1000 bootstrap replicates. The statistical significance of differences in net charge distribution between tissues of the same patient was evaluated using an unpaired t-test. Drug resistance and other mutations were identified using the Stanford HIV Drug Resistance Database (http://hivdb. stanford.edu/). Co-receptor usage prediction for sequences was performed using the web PSSM software (http://ubik.microbiol.washington.edu/computing/ pssm/).

\section{Sequence Data}

The sequence data generated in this study were deposited into GenBank under the accession numbers AY53552-AY653632 and AY874175-AY874233 for pol, and AY653633-AY653728 and AY874234-AY874280 for env sequences.

\section{Results}

\section{Virus isolation and phenotypic characterization}

We obtained a total of 80 HIV-1-infected tissue samples from 31 AIDS patients, seven tissues from three HIV-1infected asymptomatic patients, and seven tissues from six uninfected control patients (Table 1). The HIV-1-positive groups had a younger average age, and higher, more variable postmortem interval than the HIV-1-negative controls. The successful isolation of HIV-1 from these tissues correlated with the disease status and with the tis-

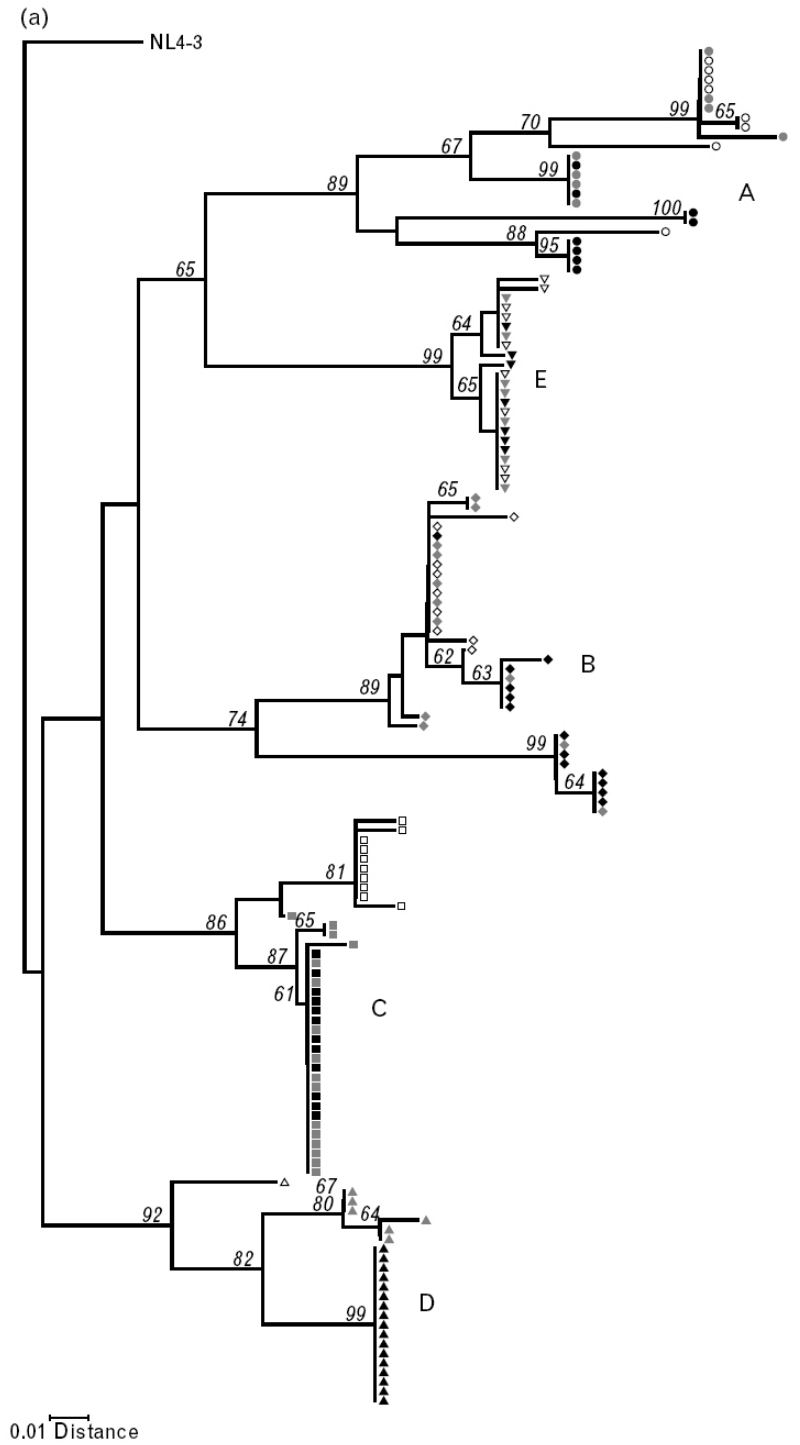

Figure 1. Phylogenetic analysis and characterization of HIV-1 env sequences. (a) Neighbor-joining phylogenetic tree (1000 bootstrapped replicates) of deduced HIV-1 env amino acid sequences (265-366 $\mathrm{HIV}_{\mathrm{HXB} 2}$ Env) isolated from brain, choroid plexus (CPx), and spleen tissues of cases A-E. Circles indicate sequences from patient $A$, diamonds indicate sequences from patient $B$, squares indicate sequences from patient $C$, triangles indicate sequences from patient $\mathrm{D}$, and inverted triangles indicate sequences from patient E. Open symbols represent brain sequences, grey symbols represent $\mathrm{CPx}$ sequences, and black symbols represent spleen sequences. Numbers on each branch indicate bootstrap values. NL4-3 (M19921) was included as an outgroup. (b-f) Alignments of deduced env amino acid sequence of molecular clones from brain $(\mathrm{Br})$, choroid plexus $(\mathrm{CPx})$, and spleen (Sp) tissues of patients A-E (panels b-f, respectively) representing residues 265-366 of $\mathrm{HIV}_{\mathrm{HXB} 2}$ Env. *Only one sequence was obtained as a result of a lack of tissue sample. \# Number of clones sequenced with the sequence indicated; periods (.) indicate gaps in the alignment, dashes (-) indicate identity with the consensus sequence, shaded sequences indicate potential $\mathrm{N}$-linked glycosylation sites. 
(b)

\#

(c)

Consensus

$\mathrm{B}-\mathrm{Br} 1$

$\mathrm{B}-\mathrm{Br} 2$

$\mathrm{B}-\mathrm{Br} 3$
$\mathrm{~B}-\mathrm{Br} 4$

$\mathrm{B}-\mathrm{Br} 4$
$\mathrm{~B}-\mathrm{Br} 5$

B-Br 5

B- CPX1

B-CPx2

$B-C P \times 2$
$B-C P \times 3$

$B-C P \times 3$
$B-C P \times 4$

B- CPx 5

$B-C P x 6$
$B-C P x 7$

B-CPx 7
B-CPx 8

B- CPX9

B- Sp1

B- Sp2

B- $\mathrm{Sp3}$

B- Sp4

B- Sp5
B- Sp6

B- Sp7

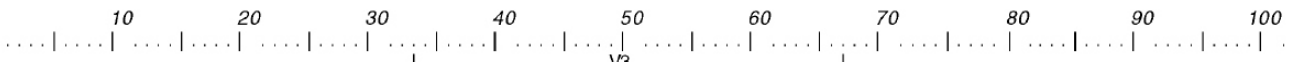

LAEDE. VVI R SDNFSDN KT IIVQL EPV II CLRPNNNT RKSI IGPGK AFYATGDIIG NIRQAHCNIS K NWNKALE QVAEKLRKQFN KTTI FKQSS G $\cdots$
1 . IIL-

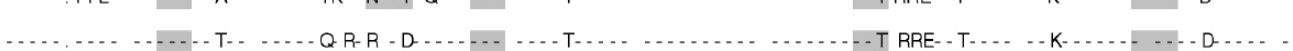
$\begin{array}{lll}\cdots & \end{array}$ $\begin{array}{lll} & \end{array}$ $\cdots$
$\cdots$ $\begin{array}{lll}\cdots & \end{array}$

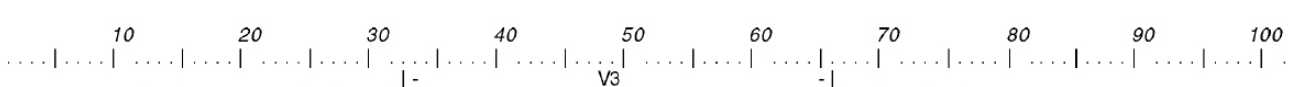

LAEEQIVI RS ENFSNNAKTI IVQLNASVEI NCTRLNNNTR RSISIGPGRA WFEAGDIIGD IRQAHCNI SK AKWNNTLRQ IVEKLRKQFG NKIIIFSSSS G 1
1 $\begin{array}{lll} & \end{array}$ $\begin{array}{llllll} & \end{array}$
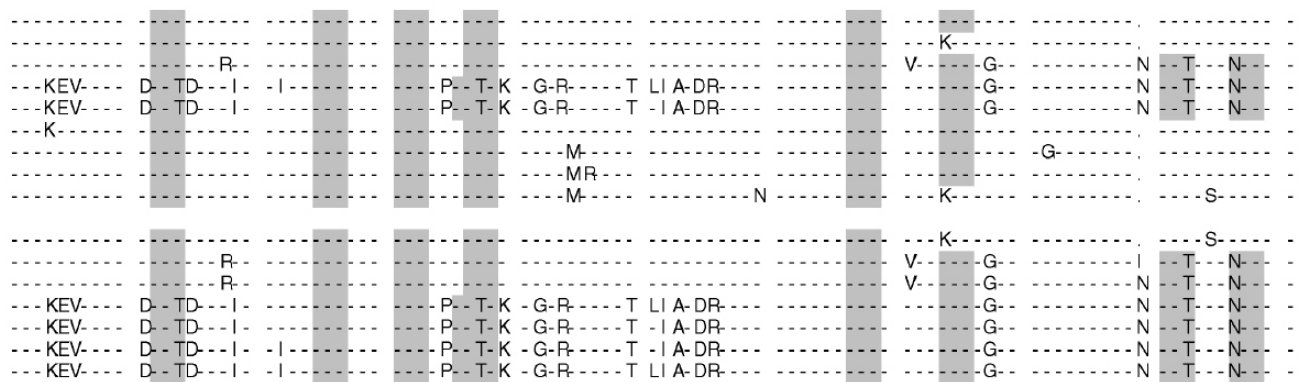

$\begin{array}{cc}\text { Glyc. } & \text { Charge } \\ 4 & +4 \\ 4 & +4 \\ 6 & +3 \\ 5 & +4 \\ & \\ 4 & +4 \\ 4 & +4 \\ 5 & +4 \\ 5 & +4 \\ & \\ 5 & +4 \\ 6 & +4 \\ 7 & +4 \\ 4 & +5\end{array}$

(d)

C.Sp1

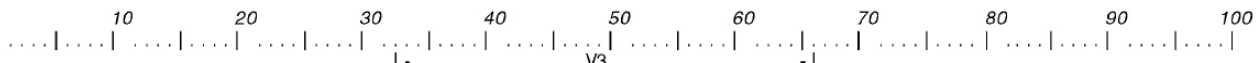
LAEKKVVIRS QNFTNNAKTI IVQLKESVEI NCTRPNNNTR KSIPIGPGRA FFATGDIIGN IRQAHCNISE AKWNNTLKQ VVKKLREQFGN KTII FNQSAG

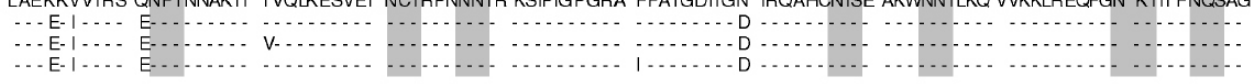

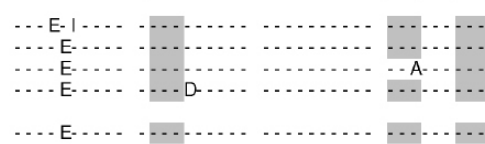

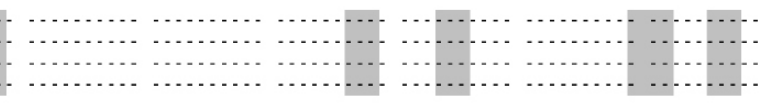

$\begin{array}{cc}\text { Glyc. } & \text { Charge } \\ 7 & +3 \\ 7 & +3 \\ 7 & +3 \\ 7 & +4 \\ 7 & +4 \\ 6 & +4 \\ 7 & +4 \\ 7 & +4\end{array}$

(e) $\ldots|\ldots|^{10} \ldots|\ldots| \ldots|\ldots| \ldots$ 30

\section{...।} $I_{\text {V3 }}^{50} \ldots|\ldots|$ .....

D-CPx1

D- $C P \times 2$

D- $C P \times 3$
D-CPx4
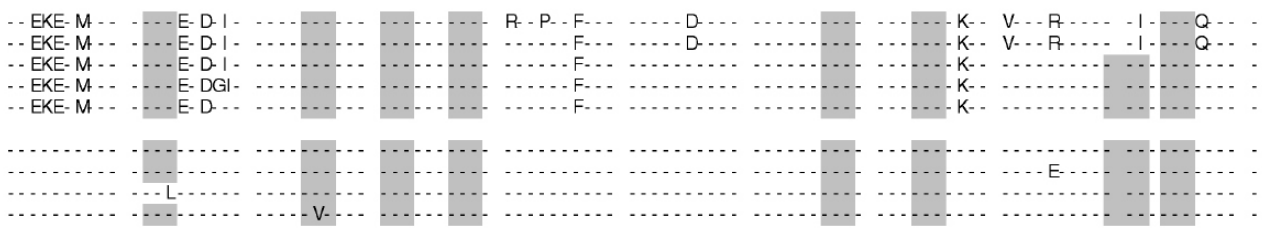

$\begin{array}{cc}\text { Glyc. } & \text { Charge } \\ 5 & +3 \\ 7 & +4 \\ 7 & +3 \\ 8 & +3 \\ 8 & +3 \\ 8 & +3 \\ 8 & +3 \\ 8 & +3 \\ 7 & +3 \\ 8 & +3\end{array}$

(f)

D-Sp4

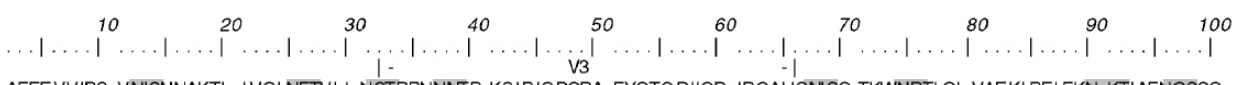

LAEEEVVIRS VNISNNAKTI IVQLNETVLI NCTRPNNNTR KSIPIGPGRA FYGTGDIIGD IRQAHCNISG TKWNRTLQL VAEKLREI FKN KTIAFNQSSG

Consensus

E-Br 1

$\mathrm{E}-\mathrm{Br} 2$

1
$\cdots$

$\mathrm{E}-\mathrm{Br} 4$
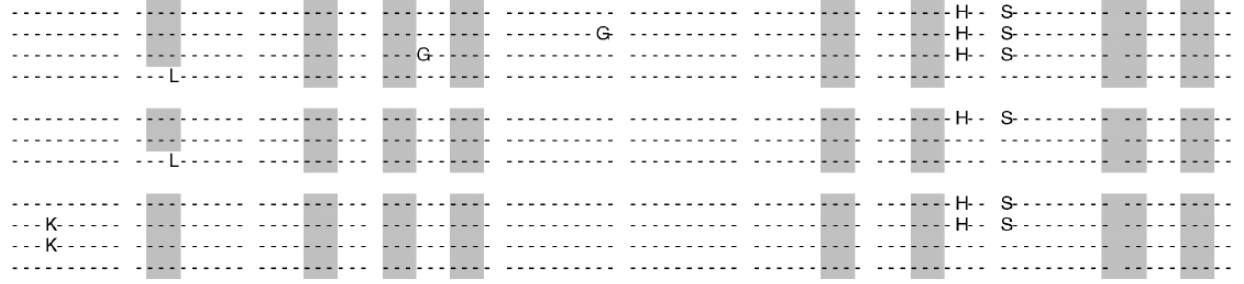

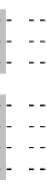

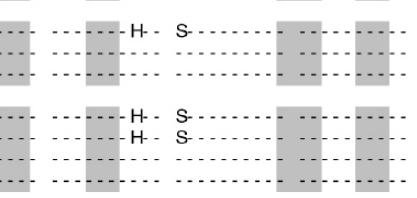

$\begin{array}{cc}\text { Glyc. } & \text { Charge } \\ 8 & +3 \\ 8 & +2 \\ 8 & +2 \\ 7 & +3 \\ 8 & +3 \\ 8 & +3 \\ 7 & +3 \\ 8 & +3 \\ 8 & +3 \\ 8 & +3 \\ 8 & +3\end{array}$

Figure 1. (continued) 
sue source, but did not correlate with HAART treatment, brain pathology, postmortem interval or PCR amplification for env sequences. Despite PCR detection of HIV-1 in a large number of samples, we successfully cultured HIV-1 in only $19.4 \%$ of the AIDS patients from at least one tissue and none from the asymptomatic group. Virus was most frequently isolated from basal ganglia $(27 \%)$, with a similar success from both $\mathrm{CPx}$ and spleen samples (18 and 12\%), and was unsuccessful from frontal or temporal cortical tissues (Table 1). Of the six cases that yielded viable virus, the neuropathological changes included: two with HIVE, (a) two with meningitis, and one each with lymphoma or white matter gliosis. The postmortem interval of these six patients averaged $10.8 \mathrm{~h}$ and ranged from 8 to $16 \mathrm{~h}$.

Among the six virus-positive cases, three had viruses isolated from all three tissues (brain, spleen, and CPx), and these isolates were further characterized (denoted A-C; Table 2). All brain and CPx virus isolates were exclusively macrophage tropic in vitro and preferentially utilized the CCR5 for viral entry. All spleen isolates were able to replicate in primary macrophages and T-cell lines, and displayed preferential usage of the CCR5 and CXCR4

\begin{tabular}{|c|c|c|c|c|c|c|c|c|c|c|c|c|c|c|c|c|c|c|c|c|c|c|c|c|c|c|}
\hline & $\mathrm{n}$ & 10 & 12 & 14 & 15 & 16 & 19 & 35 & 36 & 37 & 41 & 43 & 48 & 54 & 57 & 60 & 62 & 63 & 64 & 70 & 71 & 72 & 77 & 82 & 90 & 93 \\
\hline WT & & $\mathrm{L}$ & $\mathrm{T}$ & $\mathrm{K}$ & 1 & G & $\mathrm{L}$ & $E$ & $\mathrm{M}$ & $\mathrm{N}$ & $\mathrm{R}$ & $\mathrm{K}$ & G & 1 & $\mathrm{R}$ & D & 1 & $\mathrm{~L}$ & 1 & $\mathrm{~K}$ & A & 1 & V & V & $\mathrm{L}$ & I \\
\hline A-Brain & 9 & & & & V & & I & & 1 & & & & & & & & & ST & V & & & & & & & L \\
\hline A-CPx & 12 & & & & & & & & I & & & & & & & & & ST & V & & & $\mathrm{M}$ & & & & \\
\hline A-Spleen & 6 & & & & V & & & & 1 & & & & & & & & & ST & V & & & & & & & $\mathrm{L}$ \\
\hline B-Brain & 9 & & & & V & & & & IV & & & & & & & & V & QHR & & & & & & & & \\
\hline B-CPx & 9 & & & & V & & & & IV & & & & & & & & V & $\mathrm{H}$ & & & & & & & & \\
\hline B-Spleen & 10 & & & & $\mathrm{~V}$ & $\mathrm{E}$ & & & $\mathrm{I}$ & & & & & & & & $\mathrm{V}$ & $\mathrm{QH}$ & & & & & & & & \\
\hline C-Brain & 9 & & & $\mathrm{R}$ & & & & & & $S$ & $\mathrm{~K}$ & & & & & $\mathrm{E}$ & & $\mathrm{P}$ & & $\mathrm{T}$ & & & 1 & & & \\
\hline C-CPx & 9 & & & $\mathrm{R}$ & & & & & 1 & $S$ & $\mathrm{~K}$ & & & & & $E$ & & $P$ & & $T$ & & & 1 & & & \\
\hline C-Spleen & 9 & & & $\mathrm{R}$ & & & & & & $S$ & $\mathrm{~K}$ & & & & & $\mathrm{E}$ & & $\mathrm{P}$ & & $\mathrm{T}$ & & & 1 & & & \\
\hline D-Brain & 14 & & & & & & & $\mathrm{D}$ & & GS & $\mathrm{K}$ & & & & & & & $\mathrm{P}$ & & & & & 1 & & & L \\
\hline D-CPx & 9 & & $\mathrm{P}$ & & V & & & D & & DS & & & & & & $\mathrm{E}$ & & $\mathrm{P}$ & & & & & 1 & & & L \\
\hline D-Spleen & 7 & & $\mathrm{P}$ & & $\mathrm{V}$ & & & $\mathrm{D}$ & & $\mathrm{D}$ & & & & & & $\mathrm{E}$ & & $\mathrm{P}$ & & & & & 1 & & & L \\
\hline E-Brain & 13 & 1 & & & & & & D & I & $S$ & & 1 & V & V & $\mathrm{K}$ & & V & P & & & V & & & A & $\mathrm{M}$ & L \\
\hline E-CPx & 7 & 1 & & & & & & & 1 & $S$ & & $\mathrm{~T}$ & V & V & $\mathrm{K}$ & & V & $\mathrm{P}$ & & & V & & & A & $\mathrm{M}$ & M \\
\hline E-Spleen & 9 & 1 & & & & & & & 1 & S & & $\mathrm{T}$ & V & V & $\mathrm{K}$ & & V & $\mathrm{P}$ & & & V & & & A & $\mathrm{M}$ & M \\
\hline
\end{tabular}

(b)

\begin{tabular}{|c|c|c|c|c|c|c|c|c|c|c|c|c|c|c|c|c|c|c|c|c|c|c|c|c|c|c|c|c|c|c|c|c|c|c|c|c|}
\hline & $\mathrm{n}$ & $\frac{28}{E}$ & $\frac{35}{V}$ & $\frac{39}{\mathrm{~T}}$ & $\frac{41}{M}$ & $\frac{44}{E}$ & $\frac{60}{V}$ & $\begin{array}{r}67 \\
D\end{array}$ & $\frac{68}{\mathrm{~S}}$ & $\frac{69}{T}$ & $\frac{70}{K}$ & $\frac{75}{V}$ & $\frac{83}{R}$ & $\frac{98}{A}$ & $\frac{101}{K}$ & $\frac{103}{K}$ & $\frac{104}{K}$ & $\frac{121}{D}$ & $\frac{122}{K}$ & $\frac{2139}{T}$ & $\frac{174}{Q}$ & $\frac{177}{\mathrm{D}}$ & $\frac{7178}{1}$ & $\frac{179}{V}$ & $\frac{181}{\mathrm{Y}}$ & & $\frac{196}{G}$ & $\frac{200}{T}$ & $\frac{207}{Q}$ & $\frac{210}{L}$ & $\frac{211}{R}$ & $\frac{215}{T}$ & $\frac{219}{K}$ & $\frac{228}{L}$ & $\bar{E}$ & $\frac{250}{D}$ \\
\hline A-Brain & 9 & & & & & & I & & & & & & $\mathrm{K}$ & $\mathrm{s}$ & & & & & E & & & $\mathrm{E}$ & M & & & & $E$ & A & & & & & & & $\mathrm{N}$ & \\
\hline $\mathrm{A}-\mathrm{CPx}$ & 12 & & & & & & i & & & & & & $\mathrm{K}$ & & & & & & $\mathrm{E}$ & & & $\mathrm{E}$ & M & & & & $E$ & A & & & & & & & $\mathrm{N}$ & \\
\hline A-Spleen & 6 & & & & & & 1 & & & & & & $\mathrm{~K}$ & $\mathrm{~S}$ & & & & & $\mathrm{E}$ & & & $E$ & M & & & & $E$ & A & & & $\mathrm{K}$ & & & & & $\mathrm{N}$ \\
\hline B-Brain & 9 & & & & & & & & G & & & & & & & $\mathrm{N}$ & & & P & & $\mathrm{R}$ & $E$ & L & & & & & $E$ & $E$ & & $\mathrm{~K}$ & & $\mathrm{R}$ & & & \\
\hline B-CPx & 9 & & & & & & & & G & & & & & & & $\mathrm{N}$ & & & P & & $\mathrm{R}$ & $\mathrm{E}$ & L & & & & & E & $E$ & & $\mathrm{~K}$ & & & & & \\
\hline B-Spleen & 10 & & $\mathrm{~L}$ & & & & & & G & A & & & & & & $\mathrm{N}$ & $\mathrm{R}$ & & $\mathrm{P}$ & & $\mathrm{R}$ & $E$ & L & & & & & $E$ & $E$ & & $\mathrm{~K}$ & & & & & \\
\hline C-Brain & 9 & & & & & & I & & & & & & & & & $\mathrm{N}$ & & $\mathrm{H}$ & E & & & $\mathrm{E}$ & L & & & & & A & A & & $\mathrm{K}$ & & & $\mathrm{H}$ & & \\
\hline C-CPx & 9 & & & & & & & & & & & & & & & $\mathrm{~N}$ & & $\mathrm{H}$ & $\mathrm{E}$ & & & $\mathrm{E}$ & L & L & & & & A & A & & $\mathrm{K}$ & & & $\mathrm{H}$ & & \\
\hline C-Spleen & 9 & & & & & & & & & & & & & & & $\mathrm{~N}$ & & $\mathrm{H}$ & $\mathrm{E}$ & & & $\mathrm{E}$ & L & & & & & A & $\mathrm{T}$ & & $\mathrm{K}$ & & & & & E \\
\hline D-Brain & 14 & & L & & & & & & & & & & $\mathrm{K}$ & & & & & & & & & & & & & & $\mathrm{E}$ & & & & & & & & & \\
\hline D-CPx & 9 & & L & & & & & & & & & & $\mathrm{K}$ & & & $\mathrm{N}$ & & & & V & & & & & & & $E$ & & & & & & & & D & \\
\hline D-Spleen & 7 & & & $\mathrm{~A}$ & & & & & & & & & $\mathrm{~K}$ & & & & & & & & & & & & & & $\mathrm{E}$ & & & & & & & & & \\
\hline E-Brain & 13 & $\mathrm{~K}$ & & $\mathrm{AE}$ & L & D & I & N & & D & $\mathrm{R}$ & $M$ & & $\mathrm{~s}$ & E & $\mathrm{N}$ & & & $E$ & & & & L & & 1 & V & & & & W & & & Q & H & & \\
\hline $\mathrm{E}-\mathrm{CPx}$ & 7 & $\mathrm{~K}$ & & $\mathrm{E}$ & L & D & I & $\mathrm{N}$ & & D & $\mathrm{R}$ & M & & $\mathrm{s}$ & E & $\mathrm{N}$ & & & E & & & & & & 1 & V & & & & W & $\mathrm{K}$ & r & Q & $\mathrm{H}$ & & \\
\hline E-Spleen & 9 & $\mathrm{~K}$ & & $\mathrm{AE}$ & $\mathrm{L}$ & D & 1 & $\mathrm{~N}$ & & D & $\mathrm{R}$ & M & & $\mathrm{S}$ & $\mathrm{E}$ & $\mathrm{N}$ & & & $E$ & & & & & & 1 & $\mathrm{~V}$ & & & & W & $\mathrm{K}$ & Y & Q & $\mathrm{H}$ & & \\
\hline
\end{tabular}

Figure 2. Observed mutations and phylogenetic analysis of HIV-1 pol sequences. (a and b) The presence of relevant resistance-associated mutations at significant sites in pol for brain, choroid plexus (CPx), and spleen tissues of patients A-E compared with consensus drug-naive subtype B sequence (WT). Only those mutations that occurred in more than one clone are reported. (a) Mutations found in protease region; (b) mutations in reverse transcriptase region. (c) Neighbor-joining phylogenetic tree (1,000 bootstrapped replicates) of deduced HIV-1 pol amino acid sequences (17-418 HIV ${ }_{\text {HХB2 }}$ Pol) isolated from brain, CPx, and spleen tissues of cases A-E. Circles indicate sequences from patient $A$, diamonds indicate sequences from patient $B$, squares indicate sequences from patient $C$, triangles indicate sequences from patient $D$, and inverted triangles indicate sequences from patient E. Open symbols represent brain sequences, grey symbols represent $\mathrm{CPx}$ sequences, and black symbols represent spleen sequences. Numbers on each branch indicate bootstrap values. NL4-3 (M19921) was included as an outgroup. 


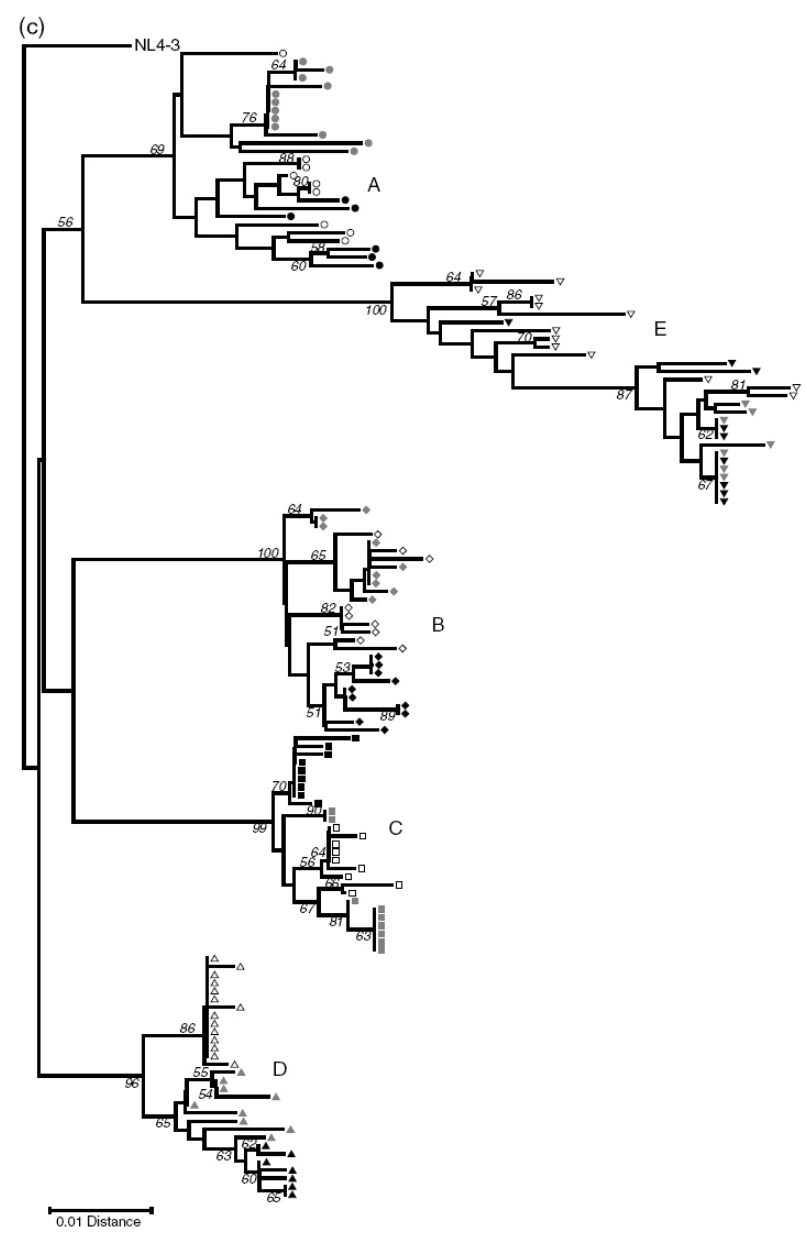

Figure 2. (continued)

co-receptors. In cross-infection experiments of CXCR4 and CCR5 co-receptor lines, we found that spleen virus was indeed a mixture of viruses rather than dual-tropic for both co-receptors. All viral isolates also exhibited multiple tissue-specific minor chemokine co-receptor usage in GHOST(3) cell lines (Table 2). The co-receptors utilized by brain viral isolates included CCR2, CCR3, CCR4 and CXCR6; all CPx isolates utilized the CCR3, CCR4, and CXCR4 co-receptors to a lesser extent than CCR5. Viral isolates from the spleen commonly utilized CCR3, CCR4, CCR6, CCR8 and CX 3 CR1.

\section{Compartmentalization of HIV-1 env}

The V3 region of env from five patients with amplifiable env sequences from three tissue compartments (Table 2) was chosen for genetic analyses because it contains sequences that are most indicative of viral tropism, especially co-receptor usage. All sequences obtained in this study were patient-specific, as sequences from each individual patient clustered together and contamination from laboratory strains was ruled out phylogenetically (Figure 1a). Each patient had env sequences that divided into at least two subgroups, indicating a level of tissue- specific variation that was discrete, but phylogenetically related (Figure 1a). Three out of five patients' brain and $\mathrm{CPx}$ sequences were more genetically similar to one another than to spleen tissue when comparisons of the average intratissue genetic distance were used (data not shown). Patient E showed no compartmentalization, but did show two distinct lineages that contained sequences from all three tissues. Generally, for HIVE-positive individuals (A, B, D, and $\mathrm{E})$, the intratissue genetic distance of $e n v$ was highest in the spleen, the number of potential N-linked glycosylation sites were reduced in brain env sequences, and the V3 loop net charge was less positive when compared with spleen $e n v$ sequences (Figures $1 \mathrm{~b}-\mathrm{d}$ ). Coreceptor usage prediction programmes found that those $\mathrm{CPx}$ sequences that showed great similarity to spleen sequences were also CXCR4-utilizing strains.

\section{Compartmentalization of viral pol}

Drug resistance mutations identified in the pol gene of viral isolates were determined by comparison with existing resistance sequences found in the Stanford drug resistance database (Figures 2a and b). Although potential drug resistance mutations were detected in tissues from all patients studied, the purpose of analysing these populations was also to examine the compartmentalization of viral pol sequences in comparison with env. As the pol gene is under strong selective pressure by antiretroviral drugs during treatment, whereas env is under immunological selective pressure, this comparison is warranted. Phylogenetic comparisons of amino acid sequences obtained from the pol of HIV-1 isolates showed a similar grouping pattern as for env (Figure 2c), but with some notable differences. Generally, pol sequences from the brain grouped together for each subject, but in some instances also showed significant similarity to spleen sequences. All five patients investigated had evidence of two pol genotype lineages that could be phylogenetically distinguished from each other, and in four out of five cases (A-D) this division was indicative of tissue compartmentalization. Furthermore, CPx virus commonly formed a unique group, but was more often related to the brain sequences than to those of peripheral origin. Interestingly, $\mathrm{CPx}$ sequences often showed extensively more $\mathrm{G} \rightarrow$ A hypermutation that resulted in the introduction of stop codons than other tissues, but these sequences were not used in subsequent analyses. Although most of the cases in this study were treated with HAART (case B history was unknown), specific details of their drug treatment histories were not available. However, based on sequence analysis of pol, all cases revealed multiple amino acid mutations that possibly conferred resistance to antiretroviral drugs (Figures $2 \mathrm{a}$ and $\mathrm{b}$ ). In addition, subject $C$ showed significant sequence differences in the pol gene from spleen virus compared with those from the brain; a relationship that is distinct from the env sequences. Other areas of hypermutation in the reverse 
transcriptase (RT) gene showed tissue-specific distribution (data not shown) but were not predictive of drug resistance.

\section{Discussion}

The CPx is a highly vascularized CNS tissue that provides a barrier between the circulatory system and the $\mathrm{CSF}$, and is responsible for the production and maintenance of CSF. Because both blood and CSF contact the surfaces of the $\mathrm{CPx}$, it is critical to understand the mechanisms whereby HIV-1 interacts with or infects the CPx. A recent report using pseudoviruses in mice [18] suggested that HIV-1 accesses the CSF via a rapid transport process, possibly transcytosis through the CPx. However, during chronic disease, CSF viral load correlates with neurological disease progression [19-21], but it is unclear whether the origin of virus found in the CSF is from resident immune cells in the CSF and associated tissues [22] or whether it is acquired from systemic sources $[19,20,23,24]$. On the basis of its function as a barrier between various compartments, including the CSF, blood, and CNS, we proposed that the CPx could play a significant role in the bidirectional dissemination of HIV between the periphery and the CNS.

We previously identified infected cells in situ and isolated HIV-1 sequences from CPx tissues [12, 25], but it was unknown whether the CPx contains productively infected cells. In the current study, we investigated the genotypic and phenotypic properties of primary HIV-1 isolates derived from the spleen, $\mathrm{CPx}$, and brain tissues. Successful viral isolation was low compared with the PCR detection of DNA sequences, possibly as a result of long postmortem intervals, low numbers of productively infected cells in the autopsy tissues, and the isolation protocols employed.

Investigation of the env and pol genes of HIV-1 in tissues from AIDS patients showed that there was clear compartmentalization of sequences in the brain compared with the periphery, but this was dependent on disease progression and the effectiveness of HAART. Although the dataset is limited to tissues from five individuals, these findings clearly demonstrate that the HIV-1 population contained within the $\mathrm{CPx}$ includes, predictably, viruses from peripheral origin, and in addition, viruses from the CNS. Specific analyses of viral sequences found in the CPx tissue showed that, overall, they are more genetically like those in the brain, but still harbor sequences similar to those in the periphery. These data are consistent with our previous study of formalin-fixed CPx tissues from four HIVE cases [12], and imply that the CPx is a tissue in which viruses or virus-infected cells from both peripheral and CNS sources can colocalize. This may be pertinent to neuropathogenesis because it is likely both to facilitate access of cytopathic or neuropathogenic variants to the CNS late in disease and to reseed archival strains from the CNS into the periphery, such as that seen in studies of patients undergoing strategic therapy interruptions of HAART who had a recurrence of drugsensitive viruses [26-29].

The pol gene, an inherently functionally conserved gene, is targeted by most antiretroviral therapies. There is strong evolutionary pressure on pol to retain function and to overcome inhibition by antiretroviral drugs, resulting in the emergence of replication-competent drugresistant viral strains. In this study of late-stage AIDS patients, there was no evidence of tissue compartmentalization of drug-resistance amino acid mutations in any of the cases studied; however, synonymous DNA polymorphisms were tissue-specific. In particular, our study shows that HIV-1 pol found in the CPx does evolve independently, despite being genetically similar to a specific tissue compartment. Disease progression is likely to influence compartmentalization, and in severe immunodeficiency, as seen in case E, there is limited compartmentalization. Regardless of this, as highlighted by specific mutations, the $\mathrm{CPx}$ seems to harbor viruses with resistance mutations similar to those found in other tissues.

Tissue compartmentalization of viral sequences occurs early, and the progenitor virus is thought to evolve independently within the unique selective microenvironment $[30,31]$. In the current study, the env gene of virus variants found in the $\mathrm{CPx}$ consisted of two genotypic lineages, indicating that there may be a cellular restriction of HIV-1 in the CPx, presumably monocytes and T cells. Our analyses of pol and env genes suggest that each gene is affected independently. As a part of the innate immunity of the CNS, the CPx possesses many cell types that can be infected by HIV-1, and thus can provide an environment for the transfer of neurocytopathic isolates to monocytes and macrophages that traffic between the CPx stroma and CSF $[32,33]$. Analysis of the phenotypic properties of HIV-1 isolated from the CPx revealed that all were non-syncytium inducing, macrophage tropic, and primarily utilized the CCR5 co-receptor for entry. Furthermore, in the two viral isolates from HIVE cases, there also existed CXCR4-tropic isolates and sequences in the CPx. As neurons and astrocytes are more vulnerable to injury and death when exposed to CXCR4-tropic than CCR5-tropic envelopes in vitro, the presence of CXCR4 strains in the CPx could contribute to brain injury $[34,35]$. In addition, genotype results support the co-localization of macrophage tropic and $\mathrm{X} 4$ cytopathic isolates in the $\mathrm{CPx}$, because some $\mathrm{CPx}$ env sequences in the $\mathrm{CPx}$ were similar to spleen sequences, and possessed a higher net V3 charge than brain sequences. This property has been associated with CXCR4 usage, T-tropism, and syncytium-inducing phenotypes [36]. Although we have previously identified HIV-1-infected monocytes 
within the CPx stroma of AIDS patients [25], future studies will be needed to identify the specific CPx cell types and viral genotypes able to infect them. Nevertheless, it is clear that the colocalization of diverse viral genotypes and phenotypes occurs within the $\mathrm{CPx}$.

The blood-CSF barrier is often overlooked in HIV-1 infection, despite its probable role in entry into the CNS. This study demonstrates that the $\mathrm{CPx}$ is a tissue compartment that contains replication-competent virus and may evolve distinct viral genotypes and phenotypes, particularly neurotoxic and drug-resistant viruses. Future investigation of the interplay between HIV-1 found in the brain, CSF, and CPx, and its relation to HAD and HIVE will clarify the significance of the CPx in AIDS neuropathogenesis.

\section{Acknowledgements}

The authors gratefully acknowledge the contributions of Ben Gelman (National NeuroAIDS Tissue Consortium at Galveston, TX, USA) for the donation of tissue specimens. The following reagents were obtained through the AIDS Research and Reference Reagent Program, Division of AIDS, NIAID, NIH: GHOST(3) panel of co-receptor expressing cell lines from Dr Vineet KewalRamani and Dr Dan Littman.

Sponsorship: These studies were supported by an NIH grant RO1 NS39177 (C.K.P. and C.W.) and an NCRR COBRE grant RR15635 (C.W.).

\section{References}

1. Pilcher CD, Shugars DC, Fiscus SA, Miller WC, Menezes P, Giner J, et al. HIV in body fluids during primary HIV infection: implications for pathogenesis, treatment and public health. AIDS 2001; 15: 837-845.

2. Delwart EL, Mullins JI, Gupta P, Learn GH Jr, Holodniy M, Katzenstein D, et al. Human immunodeficiency virus type 1 populations in blood and semen. J Virol 1998; 72: 617-623.

3. Singh A, Besson G, Mobasher A, Collman RG. Patterns of chemokine receptor fusion cofactor utilization by human immunodeficiency virus type 1 variants from the lungs and blood. J Virol 1999; 73: 6,680-6,690.

4. Haddad DN, Birch C, Middleton T, Dwyer DE, Cunningham AL, Saksena NK. Evidence for late stage compartmentalization of HIV-1 resistance mutations between lymph node and peripheral blood mononuclear cells. AIDS 2000; 14: 2,2732,281 .

5. Ohagen A, Devitt A, Kunstman KJ, Gorry PR, Rose PP, Korber B, et al. Genetic and functional analysis of full-length human immunodeficiency virus type $1 \mathrm{env}$ genes derived from brain and blood of patients with AIDS. J Virol 2003; 77: $12,336-12,345$.

6. Davis DA, Milhorat TH. The blood-brain barrier of the rat choroid plexus. Anat Rec 1975; 181: 779-789.

7. van Deurs B. Cell junctions in the endothelia and connective tissue of the rat choroid plexus. Anat Rec 1979; 195: 73-94.

8. Kivisakk P, Mahad DJ, Callahan MK, Trebst C, Tucky B, Wei $\mathrm{T}$, et al. Human cerebrospinal fluid central memory CD4+
T cells: evidence for trafficking through choroid plexus and meninges via P-selectin. Proc Natl Acad Sci USA 2003; 100: 8,389-8,394.

9. Kluge H, Hartmann W, Mertins B, Wieczorek V. Correlation between protein data in normal lumbar CSF and morphological findings of choroid plexus epithelium: a biochemical corroboration of barrier transport via tight junction pores. J Neurol 1986; 233: 195-199.

10. Petito CK. Human immunodeficiency virus type 1 compartmentalization in the central nervous system. J Neurovirol 2004; 10 (Suppl. 1): 21-24.

11. Petito CK, Chen H, Mastri AR, Torres-Munoz J, Roberts B, Wood C. HIV infection of choroid plexus in AIDS and asymptomatic HIV-infected patients suggests that the choroid plexus may be a reservoir of productive infection. J Neurovirol 1999; 5: 670-677.

12. Chen H, Wood C, Petito CK. Comparisons of HIV-1 viral sequences in brain, choroid plexus and spleen: potential role of choroid plexus in the pathogenesis of HIV encephalitis. J Neurovirol 2000; 6: 498-506.

13. Report of a Working Group of the American Academy of Neurology AIDS Task Force. Nomenclature and research case definitions for neurologic manifestations of human immunodeficiency virus-type 1 (HIV-1) infection. Neurology 1991; 41: 778-785.

14. Delwart EL, Shapaer EG, Louwagie J, McCutchan FE, Grez M, Rubsamen-Weigmann H, Mullins JI. Genetic relationships determined by a DNA heteroduplex mobility assay: analysis of HIV-1 env genes. Science 1993; 262: 1,257-1,261.

15. Lindstrom A, Albert J. A simple and sensitive 'in-house' method for determining genotypic drug resistance in HIV1. J Virol Methods 2003; 107: 45-51.

16. Delwart EL, Herring B, Rodrigo AG, Mullins JI. Genetic subtyping of human immunodeficiency virus using a heteroduplex mobility assay. PCR Methods Applications 1995; 4: 5,202-5,216.

17. Kumar S, Tamura K, Jakobsen IB, Nei M. MEGA2: molecular evolutionary genetics analysis software. Bioinformatics 2001; 17: 1,244-1,245.

18. Martin C, Albert J, Hansson P, Pehrsson P, Link H, Sonnerborg A. Cerebrospinal fluid mononuclear cell counts influence CSF HIV-1 RNA levels. J Acquir Immune Defic Syndr Hum Retrovirol 1998; 17: 214-219.

19. McArthur JC, McClernon DR, Cronin MF, Nance-Sproson TE, Saah AJ, St Clair M, Lanier ER. Relationship between human immunodeficiency virus-associated dementia and viral load in cerebrospinal fluid and brain. Ann Neurol 1997; 42: 689- 698.

20. Cinque P, Vago L, Ceresa D, Mainini F, Terreni MR, Vagani A, et al. Cerebrospinal fluid HIV-1 RNA levels: correlation with HIV encephalitis. AIDS 1998; 12: 389-394.

21. Robertson K, Fiscus S, Kapoor C, Robertson W, Schneider $\mathrm{G}$, Shepard R, et al. CSF, plasma viral load and HIV associated dementia. J Neurovirol 1998; 4: 90-94.

22. Ellis RJ, Gamst AC, Capparelli E, Spector SA, Hsia K, Wolfson $\mathrm{T}$, et al. Cerebrospinal fluid HIV RNA originates from both local CNS and systemic sources. Neurology 2000; 54: 927-936.

23. Brew BJ, Pemberton L, Cunningham P, Law MG. Levels of human immunodeficiency virus type 1 RNA in cerebrospinal fluid correlate with AIDS dementia stage. I Infect Dis 1997; 175: 963-966. 
24. Ellis RJ, Hsia K, Spector SA, Nelson JA, Heaton RK, Wallace $\mathrm{MR}$, et al. Cerebrospinal fluid human immunodeficiency virus type 1 RNA levels are elevated in neurocognitively impaired individuals with acquired immunodeficiency syndrome HIV Neurobehavioral Research Center Group. Ann Neurol 1997; 42: 679-688.

25.FalangolaMF,Hanly A, Galvao-CastroB,PetitoCK.HIV infection of human choroid plexus: a possible mechanism of viral entry into the CNS. JNeuropathol Exp Neurol 1995; 54: 497-503.

26. Verhofstede C, Noe A, Demecheleer E, De Cabooter N, Van Wanzeele F, Van Der Gucht B, et al. Drug-resistant variants that evolve during nonsuppressive therapy persist in HIV-1 infected peripheral blood mononuclear cells after long-term highly active antiretroviral therapy. J Acquir Immune Defic Syndr 2004; 35: 473-483.

27. Ryzhova EV, Crino P, Shawver L, Westmoreland SV, Lackner AA, Gonzalez-Scarano F. Simian immunodeficiency virus encephalitis: analysis of envelope sequences from individual brain multinucleated giant cells and tissue samples. Virology 2002; 297: 57-67.

28. Lafeuillade A, Khiri H, Chadapaud S, Hittinger G, Halfon $P$. Persistence of HIV-1 resistance in lymph node mononuclear cell RNA despite effective HAART. AIDS 2001; 15 : 1,965-1,969.

29. Ghosn J, Viard JP, Katlama C, de Almeida M, Tubiana R, Letourner $\mathrm{F}$, et al. Evidence of genotypic resistance diversity of archived and circulating viral strains in blood and semen of semen of pre-treated HIV-infected men. AIDS 2004; 18: $447-457$.
30. Ogert RA, Lee MK, Ross W, Buckler-White A, Martin MA, Cho MW. N-linked glycosylation sites adjacent to and within the V1/V2 and the V3 loops of dualtropic human immunodeficiency virus type 1 isolate DH12 gp120 affect coreceptor usage and cellular tropism. J Virol 2001; 75: 5,998-6,006.

31. Pollakis G, Kang S, Kliphuis A, Chalaby MI, Goudsmit J, Paxton WA. N-linked glycosylation of the HIV type-1 gp120 envelope glycoprotein as a major determinant of CCR5 and CXCR4 coreceptor utilization. J Biol Chem 2001; 276: 13,43313,441 .

32. Bragg DC, Childers TA, Tompkins MB, Tompkins WA, Meeker RB. Infection of the choroid plexus by feline immunodeficiency virus. J Neurovirol 2002; 8: 211-224.

33. Engelhardt B, Wolburg-Buchholz K, Wolburg H. Involvement of the choroid plexus in central nervous system inflammation. Microsc Res Tech 2001; 52: 112-129.

34. McCarthy M, He J, Wood C. HIV-1 strain-associated variability in infection of primary neuroglia. J Neurovirol 1998; 4: 80-89.

35. Zheng J, Thylin MR, Ghorpade A, Xiong H, Persidsky Y, Cotter $\mathrm{R}$, et al. Intracellular CXCR4 signaling, neuronal apoptosis and neuropathogenic mechanisms of HIV-1-associated dementia. J Neuroimmunol 1999; 98: 185-200.

36. De Jong JJ, De Ronde A, Keulen W, Tersmette M, Goudsmit J. Minimal requirements for the human immunodeficiency virus type $1 \mathrm{~V} 3$ domain to support the syncytium-inducing phenotype: analysis by single amino acid substitution. J Virol 1992; 66: 6,777-6,780. 